\title{
Nepetin, a Flavonoid Derived From Saussureae Involucratae, Suppresses The LPS-Induced Inflammatory Response in Cultured Human Keratinocytes
}

\section{Guowei Gong}

Zunyi Medical University

Yuzhong Zheng ( $\nabla$ zhengyuzhong@gmail.com )

Hanshan Normal University

Jian Xiao

Baoji University of Arts and Sciences

Tina Dong

Hong Kong University of Science and Technology

Karl Tsim

Hong Kong University of Science and Technology

\section{Research Article}

Keywords: Nepetin, Atopic Dermatitis, Lipopolysaccharide, Inflammatory, Keratinocytes

Posted Date: January 16th, 2021

DOI: https://doi.org/10.21203/rs.3.rs-139746/v1

License: (c) (1) This work is licensed under a Creative Commons Attribution 4.0 International License.

Read Full License 


\section{Abstract}

Background: Saussureae Involucratae Herba, known as "snow lotus" in Uyghur and/or Chinese medicines, is the dried aerial part of Saussurea involucrata (Kar. et Kir.) Sch.-Bip. (Asteraceae). One of the known pharmacological applications of this herb is to suppress chronic inflammation. Nepetin is considered as a bioactive flavonoid of Saussureae Involucratae Herba. Here, we are probing the efficacy of nepetin in modulating lipopolysaccharide (LPS)-stimulated inflammatory responses in cultured human keratinocytes.

Results: In cultured keratinocytes, applied nepetin prevented the LPS-induced cell death. In parallel, the productions of inflammatory mediators, i.e. iNOS, COX-2ХPGES2 and NO, were declined after nepetin challenge in LPS-treated keratinocytes. Besides, the treatment of nepetin in LPS-induced cultures suppressed the expressions of cytokines, i.e. IL-1 $\beta$, IL- 6 and TNF- $\alpha$, in a dose-dependent manner. The productions of inflammatory modulators and/or cytokines could be accounted by nepetin-mediated NF$\mathrm{kB}$ translocation from cytosol to nucleus within the inflammatory activated keratinocytes. In accord to this notion, the formation of ROS, as induced by LPS, could be reduced by nepetin challenge.

Conclusions: The aforementioned results suggested that nepetin could account the anti-inflammatory properties of Saussureae Involucratae Herba, at least during the skin inflammation, e.g. atopic dermatitis.

\section{Introduction}

Inflammation is commonly associated with many chronic diseases. The inflammatory process is triggered by recruitment of activated immune cells, e.g. mast cell, monocyte, macrophage and lymphocyte, to the site of lesion [1]. Skin inflammatory disorder is classified as typical dermatologic disease, categorized as acute and chronic conditions [2]. Acute disorder is commonly triggered by UV light, allergen, and physical/chemical irritants. The symptoms of acute inflammation are itching, rash and skin redness, and which are appearing and disappearing quickly [3]. On the other hand, chronic inflammation is difficult to deal with, and which could be further divided into atopic dermatitis, seborrheic dermatitis, psoriasis, and rosacea $[4,5]$. In fact, atopic dermatitis is the most common recurrent dermatological problem. Atopic dermatitis, known as atopic eczema, is caused by genetic and/or environmental factors [3], which is leading to long-term inflammation of skin, resulted in itchy, red, swollen and cracked skin. The incidence of atopic dermatitis is increasing, estimated about 230 million people suffering from this medical problem globally in 2010 [6]. Unfortunately, there is no complete cure for atopic dermatitis.

Reactive oxygen species (ROS) has been reported to play as a second messenger, and which activates immune modulatory responses [7]. Oxidative stress is the starting point, and which initiates nuclear factor kappa B (NF-kB) translocation and cytokine release [8, 9]. High levels of cytokines, e.g. interleukin 1-beta (IL-1 $\beta$ ), IL-6 and tumor necrosis factor alpha (TNF-a), as well as over expressions of inflammatory mediators, e.g. inducible nitric oxide synthase (iNOS), cyclooxygenase-2 (COX-2), prostaglandin E 
synthase 2 (PGES2) and nitric oxide (NO), aggravate the skin barrier at the injury site [10]. Corticosteroid is the standard treatment for atopic dermatitis; the usage is effective in attenuating dermatitis for shortterm application [11]. However, longer period administration of steroid may induce skin macular atrophy, striae, telangiectasia, and other side effects [12]. Therefore, the treatment of atopic dermatitis requires better and safer therapy. Several lines of evidence have suggested the possible application of flavonoidenriched herbal product in treating dermatitis $[5,6,13,14]$.

Saussureae Involucratae Herba, known as "snow lotus" in Uyghur and/or Chinese medicines, is the dried aerial part of Saussurea involucrata (Kar. et Kir.) Sch.-Bip. (Asteraceae). This herb has known efficacy in treating inflammatory-related diseases $[! 5,16]$. Nepetin is a flavonoid found in Saussureae Involucratae Herba: this flavonoid has been proposed to account for anti-cancer and anti-oxidative stress properties of the herb $[17,18]$. In human retinal pigment epithelial cells, application of nepetin decreased the phosphorylations of ERK1/2, JNK and MAPK: the $\mathrm{IC}_{50}$ of NO release was at $<7 \mu \mathrm{M}$ of nepetin $[17,19,20]$. Furthermore, nepetin inhibited asthma, induced by coal fly dust [21]. However, the potential of nepetin in treating atopic dermatitis has not been fully reported. Here, we are probing the efficacy of nepetin in modulating the lipopolysaccharide (LPS)-stimulated inflammatory responses in cultured human keratinocytes by: (i) rate of cell apoptosis; (ii) production of inflammatory mediators; (iii) release of cytokines; (iv) activation of NF-KB; and (v) formation of ROS.

\section{Methods And Materials \\ 2.1 Keratinocyte culture}

Human keratinocyte cell line, named HaCaT cell, was purchased and shipped from American Type Culture Collection (Manassas, VA) and cultured in Dulbecco's modified Eagles medium enriched with $100 \mathrm{IU} / \mathrm{mL}$ penicillin, $100 \mu \mathrm{g} / \mathrm{mL}$ of streptomycin and $10 \%$ fetal bovine serum. Cultured human keratinocyte were growth and incubated at $37{ }^{\circ} \mathrm{C}$ in a water-saturated $5 \% \mathrm{CO}_{2}$ incubator. Cells were pre-incubated with LPS $(1 \mu \mathrm{g} / \mathrm{mL})$ for 24 hours before any medical treatment. Dexamethasone (Dex, $10 \mu \mathrm{M})$ was used as a positive control in the experiments. All the chemicals were obtained from Sigma-Aldrich (St. Louis, MO).

\subsection{MTT assay}

The cell viability was measured by MTT assay (Sigma-Aldrich). In brief, cells were seeded and growth in 96-well plate. After drug treatment for 48 hours, MTT solution was added into the cultures at the final concentration of $0.5 \mathrm{mg} / \mathrm{mL}$. After incubating for 2 hours, the production of purple crystal was dissolved by DMSO. The absorbance was set at $570 \mathrm{~nm}$. Cell viability was calculated as the percentage of absorbance of blank group and value was set at 1 .

\subsection{PI-Annix staining assay}

Cells were seeded in 35-mm culture plates and allowed to grow for 24 hours in medium before drug treatment. The Annexin V-FITC/PI Apoptosis Detection kit was employed using flow cytometry (BD 
Biosciences, Franklin Lakes, NJ). Briefly, cells were washed by phosphate-buffered saline (PBS) twice and then harvested in PBS. Cells were incubated in $100 \mu \mathrm{L}$ of binding buffer, containing annexin-V/FITC and propidium iodide $(\mathrm{PI})$, for $15 \mathrm{~min}$ at room temperature in dark. The samples were automatically acquired using the loader with acquisition criteria of 10,000 events for each tube, and the quadrants were set according to the population of viable. The results were analyzed using the FlowJo v10.6 software.

\subsection{NO production}

NO Fluorometric measurement methods were performed using an Olympus Fluoview FV1000 laser scanning confocal system (Olympus America, Melville, NY), and supplied with a 10X objective. 4-amino-5methylamino-20, 70-difluorofluorescein (DAF-FM DA, Thermo Fisher Scientific, Waltham, MA) was the Intracellular NO production indicator and which was applied in our experiments. DAF-FM DA reacted not only with $\mathrm{NO}$ itself but with $\mathrm{NO}+$ equivalents, such as nitric anhydride $\left(\mathrm{N}_{2} \mathrm{O}_{3}\right)$, which were formed by autoxidation of NO. Cultured cells, seeded on the glass coverslips, after LPS stimulated for 1 day and different dosages of nepetin incubated for another 2 days, the cells were incubated for $30 \mathrm{~min}$ at $37^{\circ} \mathrm{C}$ in normal physiological solution containing $1 \mu$ M DAF-FM DM..

\subsection{Cytosolic and nuclear protein separation}

After drug presence for 48 hours in cultured cells, 1X PBS was used to wash the cultures, and then using Qproteome Nuclear Protein Kit (Qiagen, Francisco, CA) to separate nuclear and cytosol NF-kB protein. After that, the samples were dissolved in lysis buffer $(0.125 \mathrm{M}$ Tris- $\mathrm{HCl}, \mathrm{pH} \mathrm{6.8,4 \%} \mathrm{SDS,} \mathrm{20 \%} \mathrm{glycerol,}$ and 2\% 2-mercaptoethanol) for Western blot assay.

\subsection{Western blot and phosphorylation}

Cell lysate were subjected to SDS-PAGE in revealing protein expresstional levels of target genes. After transferring the target proteins to membranes, the membranes were incubated with anti-iNOS (CST, Danvers, MA) at 1:5,000 dilutions, anti-COX-2 (CST) at $1: 5,000$ dilutions, anti-PGES2 (Santa Cruz, Dallas, TX) at 1:5000 dilutions, anti-NF-kB (p65) (CST) at $1: 5,000$ dilutions, anti-lkBa (CST) at $1: 5,000$ dilutions, anti-histon-1 (CST) at 1:5,000,000, anti- $\beta$-actin (CST) at $1: 5,000$ dilutions and anti-GAPDH (Santa Cruz) at $1: 5,000,000$ dilutions at $4{ }^{\circ} \mathrm{C}$ for overnight. Following incubation in horseradish peroxidase (HRP)conjugated secondary antibodies for 3 hours at room temperature, the immune-complexes were visualized by the enhanced chemiluminesence (ECL) method (Amersham Biosciences, Piscataway, NJ). The band intensities were calculated and analyzed by Image $\mathrm{J}$.

\subsection{NF-kB translocation assay}

The translocation of p65 was revealed with Olympus Fluoview FV1000 confocal system (Olympus America) mounted on an inverted Olympus microscope (63X objective). The antibodies for $p 65$ and $\beta$ actin were diluted in cold PBS containing $2.5 \%$ fetal bovine serum and $0.1 \%$ Triton X-100 (Sigma-Aldrich) after cell fixed, using $4 \%$ methanol-free paraformaldehyde treatment for $10 \mathrm{~min}$. Samples were kept in cold room for overnight, and then 1:1,000 dilution of FITC-labeled anti-rabbit antibody (Jackson Laboratories, West Grove, PA) was used as secondary antibody, incubated in dart at room temperature for 
3 hours. After incubating, the cells were washed for 3 times and. DAPI (1: 5,000 dilution) was used to stain nucleus before performing assay.

\subsection{ROS formation}

Fluorometric measurements of ROS were utilized CellROX Deep Red Fluorescence Flow Cytomer detection kit (BD Biosciences) under manufacturer's suggested protocol. Briefly, cells at were incubated in $100 \mu \mathrm{L}$ of binding buffer for $15 \mathrm{~min}$ at room temperature. Samples were automatically acquired using the loader with acquisition criteria of 10,000 events for each tube, and the quadrants were set according to population of viable. The cytometer results were analyzed with Flowjo v7.6 software.

\subsection{Chromatography}

The chromatography of methanol extract of Saussureae Involucratae Herba was described by Gong et al. (2020b). Agilent 1200 series system (Agilent, Waldbronn, Germany), supplied with degasser, binary pump, auto-sampler and thermo-stated column compartment was utilized. Chromatographic run was carried out on an Agilent, Eclipse Plus, C18 column $(4.6 \times 250 \mathrm{~mm}, 5 \mu \mathrm{m})$ with acetonitrile (as solvent A) and $0.01 \%$ formic acid (as solvent B) in the mobile phase at a flow rate of $1.0 \mathrm{~mL} / \mathrm{min}$. Ten $\mu \mathrm{L}$ sample or nepetin marker (Sigma-Aldrich) were injected.

\subsection{Statistical analyses and other assays}

Protein concentrations were calculated measured by Bradford protein assay dye from Bio-Rad (Bio-Rad Laboratories, Hercules, CA). The protein sample was collected from cell lysis. Then, the aliquot of sample or different doses of BSA standard was mixed with assay dye by gently shaking for 1 min followed by measured at $595 \mathrm{~nm}$ using Multiskan ${ }^{\mathrm{TM}}$ FC Microplate Photometer. The protein concentration was calculated according to the standard curve. Each result is represented as the Mean \pm SEM. Comparisons of the means for untreated control cells and treated cells were analyzed using one-way ANOVA (Bonferroni's post-test). Significant values were indicated by ${ }^{*} p<0.05,{ }^{* *} p<0.01$ and ${ }^{* \star *} p<0.001$ as compared to the blank group. Significant values were indicated by ${ }^{\wedge} p<0.05,{ }^{\wedge}{ }^{\wedge} p<0.01$ and ${ }^{\wedge \wedge}{ }^{\wedge} p<0.001$ as compared to the LPS group.

\section{Results}

\subsection{Nepetin decreases LPS-induced responses}

Prior conducting biological analysis, the purity of nepetin, determined by HPLC, and the maximal working concentration of nepetin in cultured cells, determined by MTT analysis, were calibrated. Nepetin below 10 $\mu \mathrm{M}$ did not show toxicity to cultured keratinocytes (Supplementary Fig. 1), and therefore safety concentration of nepetin was used here. From the results, the purity of nepetin was higher than $98 \%$, and which could be utilized for the following assays (Supplementary Fig. 1). The inhibition of cell apoptosis was analyzed in LPS-induced cultured HaCaT cells in the presence or absence of nepetin. Annexin VFITC- and PI-labeled keratinocytes were subjected to flow cytometry analysis. HaCaT cell viability was 
dramatically altered in the presence of LPS at $1 \mu \mathrm{g} / \mathrm{mL}$ (Fig. 1A\&B). The dose of LPS was in accord to the published reports $[8,9,17]$. The LPS at $1 \mu \mathrm{g} / \mathrm{mL}$ induced apoptotic cell to $\sim 12$-fold increase, as compared to the control group, i.e. inducing cell death to $\sim 60 \%$ of total cell population (Fig. 1B). Therefore, LPS at $1 \mu \mathrm{g} / \mathrm{mL}$ was applied for the following experiments. Dexamethasone at $10 \mu \mathrm{M}$ prevented the LPS-induced cell death significantly to $~ 20 \%$ of total (Fig. 1A\&B). Nepetin at different doses decreased the LPSinduced HaCaT cell apoptosis in a dose-dependent manner (Fig. 1A\&B). In the presence of $3 \mu \mathrm{M}$ nepetin in LPS-induced cells, the apoptotic rate was deceased to 50\%, as compared to the LPS-treated group (Fig. 1B).

Skin keratinocyte is the first line of defensive barrier against external stimulus, and which triggers immune responses of skin by producing inflammatory mediators and cytokines. In cultured HaCaT cells, the treatment of LPS stimulated the expressions of iNOS, COX-2 and PGES2, as compared to the blank control (Fig. 2A). LPS triggered the levels of inflammatory mediators to $\sim 2.5$-fold for iNOS, $\sim 5$-fold for COX-2 and $~ 4.5$-fold for PGES2. To examine the potential anti-inflammatory properties of nepetin on LPS-induced dermatitis in cultured HaCaT cells, nepetin was applied onto the culture for 48 hours. The expression levels of iNOS, PGES2 and COX-2 were declined after nepetin treatment in the LPS-treated cultures: this suppression was in a dose-dependent manner (Fig. 2A). Nepetin at $3 \mu \mathrm{M}$ showed the highest suppression on the protein expressions. Moreover, the increased production of NO, induced by LPS, in cultured keratinocytes was abolished by nepetin in a dose-dependent manner, as revealed by DAF-FM fluorescence (Fig. 2B). Nepetin at $3 \mu \mathrm{M}$ restricted the LPS-induced NO production to $\sim 60 \%$.

Dexamethasone was a positive control, and which suppressed the LPS-mediated inflammatory responses back to the blank control (Fig. 2A\&B).

The amounts of cytokines, i.e. IL-1 $1 \beta, \mathrm{IL}-6$ and TNF- $a$, in cultured keratinocytes after exposure of LPS were measured. LPS induced the production of cytokines significantly, at least over 10-folds, in dosedependent manners (Fig. 3). Flow cytometer data showed that the production of IL-1 $\beta$ was significantly down-regulated by nepetin in LPS-treated keratinocyte, as compared with LPS model group (Fig. 3A). Three $\mu \mathrm{M}$ of nepetin reduced the IL- $1 \beta$ production to $\sim 30 \%$ of the LPS-treated group. Similar scenarios were observed for the releases of IL- 6 and TNF- $a$ in exposing to nepetin for 48 hours in the LPSstimulated keratinocytes (Fig. 3B\&C).

\subsection{Nepetin mitigates NF-KB translocation and ROS formation}

The induction of ROS formation and NF-KB signaling have been reported to be involved in the pathogenesis of atopic dermatitis. Agents having the activity of modulating ROS formation and maintaining NF-KB transduction have been considered to use in treating inflammatory skin diseases [7, 22]. NF-KB is an ubiquitous transcription factor, and the primary role is responsible to mediate inflammatory stimuli. The inactivated NF-KB, located in cytosol, is being complexed with an inhibitory protein $\mathrm{IKBa}$. Once being stimulated, IKBa undergoes degradation and release of NF-KB migrating to nucleus $[8,16]$. The translocation of NF-KB was noted in cultures with LPS (Fig. 4). Immunostaining 
results indicated that the present of LPS could induce NF-KB p65 translocation from cytosol to nucleus

(Fig. 4). Dexamethasone suppressed the translocation of p65 in LPS-induced keratinocytes. The exposure of nepetin in LPS-treated cultures, dose-dependently, modified the translocation of p65 (Fig. 4). To further confirm the p65 activity, the amounts of p65 within separated cytosolic and nucleus fractions were determined by western blotting. The expression of p65 in nucleus was increased by applied LPS in cultured keratinocytes (Fig. 5A). Application of nepetin was able to suppress the p65 translocation, induced by LPS, in the nucleus: this effect was in a dose-dependent manner. As expected, the positive control dexamethasone showed similar suppression. In parallel, the level of NF-kB in cytosolic fraction was measured. Contrasting to nucleus, LPS reduced the level of NF-kB in cytosol, and this reduction was partially restored by application of nepetin in cultured keratinocytes, as in a dose-dependent manner (Fig. 5B). Moreover, the protein expression level of IkBa in cytosol was determined, and which was reduced by LPS application. Nepetin, or dexamethasone, could restore the reduction, at least partially (Fig. 5B).

The inhibition of ROS generation has been reported to be important in preventing dermal tissue damage during skin barrier infection and inflammation $[10,11]$. Having this notion, the ROS formation in the LPSstimulated keratinocytes, with or without nepetin, was measured. Application of LPS markedly induced the formation of ROS, at least over 12-fold of activation (Fig. 6). The treatment of nepetin, dosedependently, suppressed the LPS-induced ROS formation in a concentration-dependent manner (Fig. 6A\&B). The ROS formation under dexamethasone showed neutralization effect in the LPS-treated keratinocytes.

\section{Discussion}

Flavonoids have a benzo-y-pyrone basic structure and consist of myriad polyphenolic compounds. Flavonoids work as secondary metabolites by encouraging plant to adapt to environment, producing pigmentation and protecting the plant against pathogens [23]. Flavonoid is a multi-functional phytochemical presenting substantial characteristics that can be exploited for therapeutic agents targeting on various diseases, pharmaceutically. Flavonoids are believed as pivotal components for daily supplement [23]. Various flavonoid-enriched functional foods have been reported to have an antiinflammatory function, e.g. Astragali Radix, green tea, Ginkgo Folium [24]. Studies have demonstrated that theses functional foods perform anti-inflammatory properties by blocking the inflammatory mediators, e.g. NO, COX-2, TNF-a, IL-1 $\beta$, IL-6, IL-15 and interferon- $\gamma$ [25]. Therefore, these functional foods are likely to modulate TLRs, NF-KB, AP-1, and IRFs inflammatory signaling pathways, as well as to suppress intracellular inflammatory signaling molecules [25].

The categorization of flavonoids is divided into six major subclasses, namely anthocyanins, flavan-3-ols, flavanones, flavones, flavonols, and isoflavones [23]. Nepetin is a natural flavone. The immunemodulatory functions of nepetin are documented. Chen et al. (2018) reported inhibitory effect of nepetin in retinal pigment epithelial cells (ARPE- 19 cells) by down-regulating IL- $1 \beta$ via altering NF-KB and MAPK signaling pathways in regulating retinal diseases [17]. The presence of nepetin attenuated COX-1 and 
COX-2 productions in cultured RAW 264.7 cells after 24 hours of treatment [26]. Furthermore, Ji et al. (2020) found that nepetin suppressed degranulation and generation of leukotriene C4 and inhibited prostaglandin D2 in antigen-stimulated bone marrow derived mast cells by blocking Akt and NF-KB transduction [18].

Inflammation is critical in responding to infection or pathogen by activating expression of cytokine and/or inflammatory mediator, aiming to recruit T-cells and myeloid cells [5]. The inflammatory mediators, i.e. NO, has been confirmed to be monitored by inducible isoforms of iNOS and COX-2 during inflammation [27]. Abnormal production of inflammatory mediators has been noticed after external stimulus: this abnormality is associated with various immune-related diseases, as well as tumorigenesis in different cell types [9]. Several lines of evidence have reported that atopic dermatitis is closely associated with inflammation [28, 29]. LPS, a well-studied external stimulus, leads to accelerate apoptotic rate of human keratocytes, as well as to trigger production of inflammatory mediators [30]. Here, we have shown that an application of nepetin in cultured keratinocytes could reduce LPS-induced cell death. Moreover, nepetin inhibited LPS-induced expression/production of iNOS, COX-2, PGES2 and NO. Thitilertdecha et al. (2019) found that nepetin exhibited suppressive function on early T-cell activation by altering cell death rate [31]. Makino et al. (2008) reported that the herbal formulae mitigated atopic dermatitis-like symptoms in skin, and the herbal treatment decreased the expressions of IL-4 and IFN- $\gamma$ mRNAs [32]. Interestingly, these herbal formulae all contained nepetin as major chemical. Taken together, the aforementioned results suggest that nepetin, or its parental herb Saussureae Involucratae Herba, has the potential pharmaceutical value in treating skin atopic dermatitis.

Chinese herbal medicines have been well-recognized clinically and widely recommended for treatment of atopic dermatitis [33,34]. However, there is no scientific evidence, or proposed action mechanism, to support the utilization of herbal medicine, and therefore patients and/or consumers are still concerned about the efficacy and safety [35]. Bioactive agents identified and isolated from natural resources have drawn great attention with precise mechanism and lower side effects, as compared to the synthesized medicine. Therefore, herbal medicine is playing critical function in drug discovery. Having an increase usage of herbal products in clinics for atopic dermatitis, active chemicals have been isolated for such treatment, e.g. methoxsalen isolated from Ammi majus and capsaicin isolated from chill pepper [36]. Despite nepetin has shown positive function in responding to inflammatory progression in vitro, animal and more controlled clinical data are required to further confirm the efficacy and safety of this plantisolated chemical in dermatology.

\section{Abbreviations}

COX-2: Cyclooxygenase-2; DAF-FM DA: 4-amino-5-methylamino-20, 70-difluorofluorescein;

ECL: Enhanced chemiluminesence; IL: Interleukin; iNOS: Inducible nitric oxide synthase; LPS:

Lipopolysaccharide; NF-kB: Nuclear factor kappa B; NO: Nitric oxide; PBS: Phosphate-buffered saline; 
PGES2: Prostaglandin E synthase 2; PI: Propidium iodide; ROS: Reactive oxygen species; TNF-a: Tumor necrosis factor alpha;

\section{Declarations}

\section{Ethics approval and consent to participate}

Not applicable

\section{Consent for publication}

Not applicable

\section{Availability of data and materials}

The datasets used and/or analyzed during the current study are available from the corresponding author on reasonable request.

\section{Competing interests}

The authors declare that they have no competing interests.

\section{Conflict of Interest}

The author(s) declare(s) that there is no conflict of interests regarding the publication of this paper.

\section{Funding}

Supported by Research Fund of Guizhou Provincial Natural Science Foundation, QKH-J [2020] 1Y377, Zunyi Science and Technology Project [ZSKHHZZ(2020)85], Science and Technology Program of Guizhou Province (QKPTRC[2019]-024) Zunyi Medical University for the Doctoral Program (F-937), Natural Science Foundation of Guangdong Province (2018A030307074 and 2019-GDXY-0031), Shenzhen Science and Technology Innovation Committee (ZDSYS201707281432317; JCYJ20180214151016621; JCYJ20180306174903174; JCYJ20170413173747440; JCYJ20160229205812004; JCYJ20160229210027564; CKFW2016082916015476), Guangzhou Science and Technology Committee Research Grant (GZSTI16SC02; GZSTI17SC02), Hong Kong RGC Themebased Research Scheme (T13-607/12R), Hong Kong Innovation Technology Fund (UIM/385, ITS/500/18FP; TCPD/17-9) and HMRF18SC06.

\section{Authors and Contributors}

Guowei Gong, Yuzhong Zheng and Karl Tsim wrote the main text. Guowei Gong was responsible for the experiments. Jian Xiao helped reagent preparation. Tina TX Dong was responsible for purchasing reagents and chemicals. All authors read and approved the final version of manuscript. 
Acknowledgements

Not applicable.

\section{References}

1. Singh N, Baby D, Rajguru J, Patil P, Thakkannavar S, Pujari V. Inflammation and cancer. Ann Afr Med 2019; 18: 121-126

2. Waldman AR, Ahluwalia J, Udkoff J, Borok JF, Eichenfield L.F. Atopic dermatitis. Pediatr Rev 2018; 39: 180-193.

3. Berke R, Singh A, Guralnick M. Atopic dermatitis: an overview. Am Fam Physician 2012; 86: 35-42.

4. Hussain Z, Thu HE, Shuid AN, Kesharwani P, Khan S, Hussain F. Phytotherapeutic potential of natural herbal medicines for the treatment of mild-to-severe atopic dermatitis: a review of human clinical studies. Biomed Pharmacother 2017; 93: 596-608.

5. Hon KL, Leung AKC, Leung TNH, Lee VWY. Investigational drugs for atopic dermatitis. Expert Opin Investig Drugs 2018; 27: 637-647.

6. Nygaard U, Vestergaard C, Deleuran, M. Emerging treatment options in atopic dermatitis: systemic therapies. Dermatology 2017; 233: 344-357.

7. Sivaranjani N, Rao S.V, Rajeev G. Role of reactive oxygen species and antioxidants in atopic dermatitis. J Clin Diagn Res 2013; 7: 2683-2685.

8. Gong AG, Zhang LM, Lam CT, Xu ML, Wang HY, Lin HQ, Dong TT, Tsim KW. Polysaccharide of Danggui Buxue Tang, an ancient Chinese herbal decoction, induces expression of pro-inflammatory cytokines possibly via activation of NFKB signaling in cultured Raw 264.7 cells. Phytother Res 2017; 31: 274-283.

9. Gong G, Wang H, Kong X, Duan R, Dong TTX, Tsim KWK. Flavonoids are identified from the extract of Scutellariae Radix to suppress inflammatory-induced angiogenic responses in cultured RAW 264.7 macrophages. Sci Rep 2018; 8: 17412.

10. Choi D, Kang W, Park T. Anti-allergic and anti-inflammatory effects of undecane on mast cells and keratinocytes. Molecules 2020; 25: 1554.

11. Perkins MA, Osterhues MA, Farage MA, Robinson MK. A noninvasive method to assess skin irritation and compromised skin conditions using simple tape adsorption of molecular markers of inflammation. Skin Res Technol 2001; 7: 227-237.

12. Hobbs RM, Silva-Vargas V, Groves R, Watt FM. Expression of activated MEK1 in differentiating epidermal cells is sufficient to generate hyperproliferative and inflammatory skin lesions. $J$ Invest Dermato/ 2004; 123: 503-515.

13. Lee JH, Jo EH, Lee B, Noh HM, Park S, Lee YM, Kim DK, Park MC. Soshiho-Tang, a traditional herbal medicine, alleviates atopic dermatitis symptoms via regulation of inflammatory mediators. Front Pharmacol 2019; 10: 742. 
14. Kang SJ, Jo EH, Yang GJ, Shim YH, Hong JE, Park MC. Efficacy and safety of Soshiho-tang in patients with atopic dermatitis and gastrointestinal disorders: study protocol for a double-blind, randomized, and placebo-controlled clinical trial. Medicine (Baltimore) 2019; 9:

15. Gong G, Huang J, Yang Y, Qi B, Han G, Zheng Y, He H, Chan K, Tsim K.W, Dong TT. Saussureae Involucratae Herba (snow lotus): review of chemical compositions and pharmacological properties. Front Pharmacol 2020a; 10: 1549.

16. Gong G, Xie F, Zheng Y, Hu W, Qi B, He H, Dong TT, Tsim KW. The effect of methanol extract from Saussurea involucrata in the lipopolysaccharide-stimulated inflammation in cultured RAW 264.7 cells. J Ethnopharmaco/ 2020b; 251: 112532

17. Chen X, Han R, Hao P, Wang L, Liu M, Jin M, Kong D, Li X. Nepetin inhibits IL-1 $\beta$ induced inflammation via NF-KB and MAPKs signaling pathways in ARPE-19 cells. Biomed Pharmacother 2018; 101: 87-93.

18. Ji N, Kim SG, Park HH, Lee E, Lee YJ, Jin M, Lee E. Nepetin, a natural compound from Inulae Flos, suppresses degranulation and eicosanoid generation through PLCY1 and Akt signaling pathways in mast cells. Arch Pharm Res 2020; 43: 224-232.

19. Clavin M, Gorzalczany S, Macho A, Muñoz E, Ferraro G, Acevedo C, Martino V. Anti-inflammatory activity of flavonoids from Eupatorium arnottianum. J Ethnopharmacol 2007; 112: 585-589.

20. Garayev E, Di GC, Herbette G, Mabrouki F, Chiffolleau P, Roux D, Sallanon H, Ollivier E, Elias R, Baghdikian B. Bioassay-guided isolation and UHPLC-DAD-ESI-MS/MS quantification of potential anti-inflammatory phenolic compounds from flowers of Inula montana L. J Ethnopharmaco/ 2018; 226: 176-184.

21. Saba E, Lee YS, Yang WK, Lee YY, Kim M, Woo SM, Kim K, Kwon YS, Kim TH, Kwak D, Park YC, Shin HJ, Han CK, Oh JW, Lee YC, Kang HS, Rhee MH, Kim SH. Effects of a herbal formulation, KGC3P, and its individual component, nepetin, on coal fly dust-induced airway inflammation. Sci Rep 2020; 10 : 14036.

22. Niwa $Y$, Sumi H, Kawahira K, Terashima T, Nakamura T, Akamatsu H. Protein oxidative damage in the stratum corneum: evidence for a link between environmental oxidants and the changing prevalence and nature of atopic dermatitis in Japan. Br J Dermato/ 2003; 149: 248-254.

23. Panche AN, Diwan AD, Chandra SR. Flavonoids: an overview. J Nutr Sci 2016; 5: e47.

24. Kumar S, Pandey A. Chemistry and biological activities of flavonoids: an overview. Sci World J 2016;

25. Chen AY, Chen Y. A review of the dietary flavonoid, kaempferol on human health and cancer chemoprevention. Food Chem 2013; 138: 2099-2107.

26. Lajter I, Pan SP, Nikles S, Ortmann S, Vasas A, Csupor-Löffler B, Forgó P, Hohmann J, Bauer R. Inhibition of COX-2 and NF-KB1 gene expression, NO production, 5-LOX, and COX-1 and COX-2 enzymes by extracts and constituents of Onopordum Acanthium. Planta Med 2015; 81: 1270-1276.

27. Posadas I, Terencio MC, Guillén I, Ferrándiz ML, Coloma J, Payá M, Alcaraz MJ. Co-regulation between cyclo-oxygenase-2 and inducible nitric oxide synthase expression in the time-course of murine inflammation. Naunyn Schmiedebergs Arch Pharmacol 2000; 361: 98-106. 
28. Cabanillas B, Brehler AC, Novak N. Atopic dermatitis phenotypes and the need for personalized medicine. Curr Opin Allergy Clin Immunol 2017; 17: 309-315

29. Furue M, Kadono, T. "Inflammatory skin march" in atopic dermatitis and psoriasis. Inflamm Res 2017; 66: 833-842.

30. Yan H, Wang Y, Shen S, Wu Z, Wan P. Corticosteroids effects on LPS-induced rat inflammatory keratocyte cell model. PLoS One 2017; 12: e0176639.

31. Thitilertdecha P, Tantithavorn V, Poungpairoj P, Onlamoon N. Determination of suppressive effect on human T-cell activation by hispidulin, nepetin, and vanillic acid. Immunopharmacol Immunotoxicol 2019; 41: 591-598.

32. MakinoT, Hamanaka M, Yamashita H, Mizukam H. Effect of bakumijiogan, an herbal formula in traditional Chinese medicine, on atopic dermatitis-like skin lesions induced by mite antigen in NC/Jic mice. Biol Pharm Bull 2008; 31: 2108-2113.

33. Kim HI, Hong SH, Ku JM, Kang S, Kim TY, Shin YC, Ko SG. Tonggyu-tang, a traditional Korean medicine, suppresses pro-inflammatory cytokine production through inhibition of MAPK and NF-KB activation in human mast cells and keratinocytes. BMC Complement Altern Med 2017; 17: 186

34. Wu PC, Chuo WH, Lin SC, Lehman CW, Lien CZ, Wu CS, Lin CC. Sclareol attenuates the development of atopic dermatitis induced by 2,4-dinitrochlorobenzene in mice. Immunopharmacol Immunotoxicol 2019; 41: 109-116.

35. Lin HQ, Gong AG, Wang HY, Duan R, Dong TT, Zhao KJ, Tsim KW. Danggui Buxue Tang (Astragali Radix and Angelicae Sinensis Radix) for menopausal symptoms: a review. J Ethnopharmaco/ 2017; 199: 205-210.

36. Reuter J, Merfort I, Schempp CM. Botanicals in dermatology: an evidence-based review. Am J Clin Dermato/ 2010; 11: 247-267.

\section{Figures}




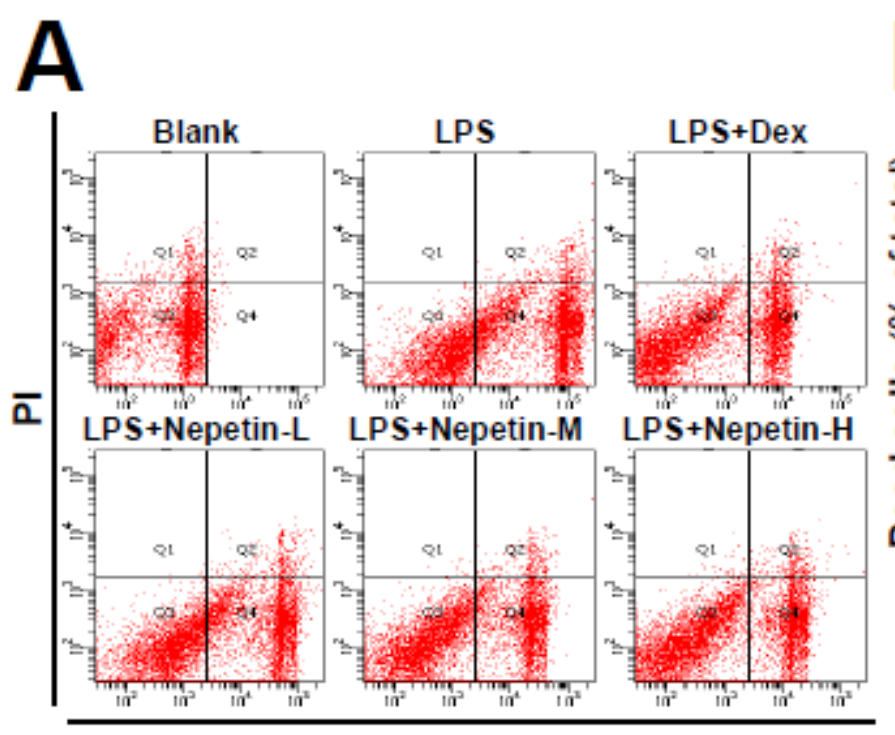

Annexin V FITC

\section{B}

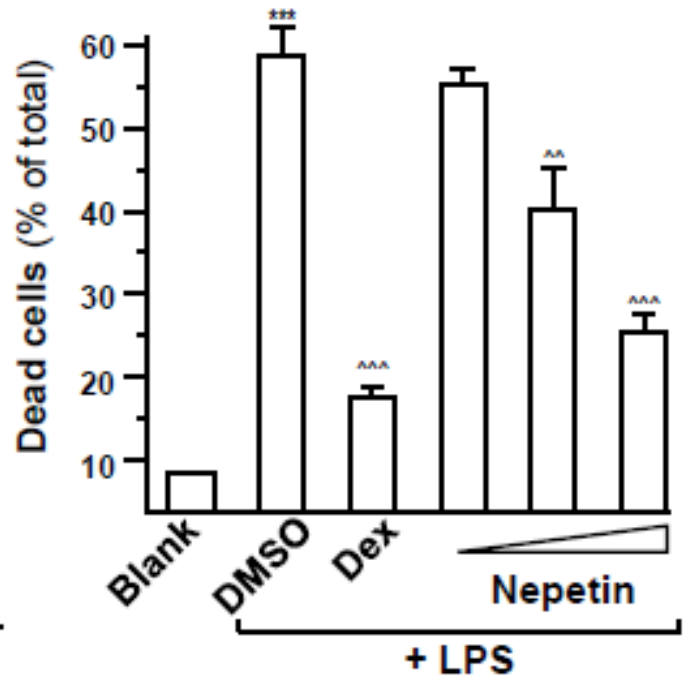

\section{Figure 1}

Nepetin reduces LPS-induced cell death Cell apoptosis was detected by Annexin V/PI apoptosis detection kit. [A]: Cultured HaCaT was treated with LPS $(1 \mu \mathrm{g} / \mathrm{mL})$ for 24 hours and then applied with nepetin for another 48 hours $(0.3 \mu \mathrm{M}$ at $\mathrm{L}, 1 \mu \mathrm{M}$ at $\mathrm{M}$ and $3 \mu \mathrm{M}$ at $\mathrm{H}$, as indicated). The dual parametric dot plots combining annexin V-FITC and PI fluorescence showed the viable cell population in the bottom left quadrant (Q3), the early apoptotic cells in the bottom right quadrant (Q4), and the late apoptotic cells in the top right quadrant (Q2). [B]: The quantification analysis was performed by Flowjo v7.6 software. Dexamethasone (Dex) at $10 \mu \mathrm{M}$ was served as a positive control. The values are expressed as percentage of total cell number, in Mean \pm SEM. ${ }^{\star \star \star} p<0.001$ was considered as significant result compared to the blank group (no drug). ${ }^{\wedge} \mathrm{p}<0.01$ and ${ }^{\wedge \wedge \wedge} \mathrm{p}<0.001$ were categorized as significant result compared to the LPS group. 

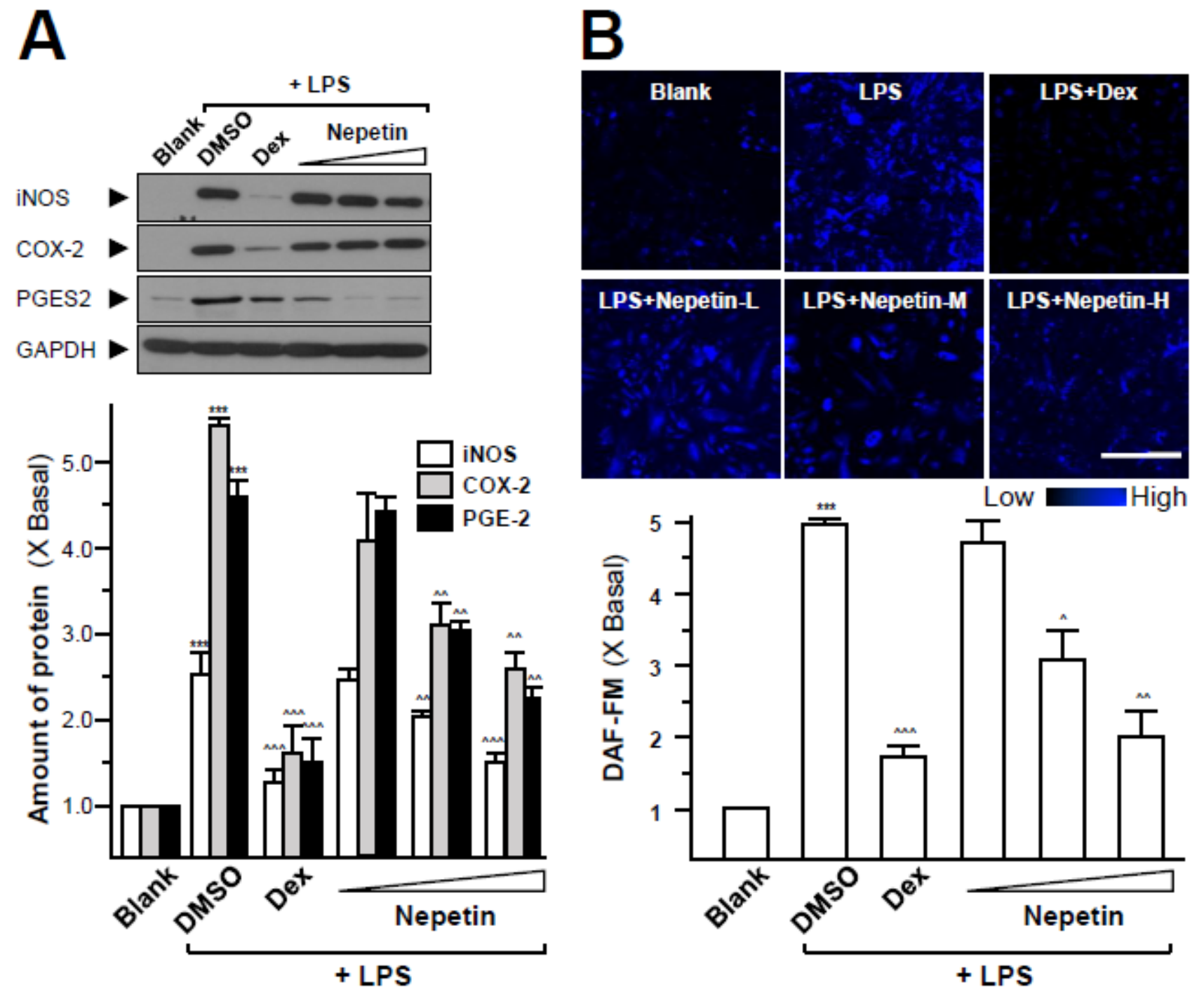

Figure 2

Nepetin suppresses inflammatory mediators' productions The LPS $(1 \mu \mathrm{g} / \mathrm{mL})$-pretreated cultured HaCaT cells were treated with nepetin ( $0.3 \mu \mathrm{M}$ as $\mathrm{L}, 1 \mu \mathrm{M}$ as $\mathrm{M}$ and $3 \mu \mathrm{M}$ as $\mathrm{H}$, as indicated) or dexamethasone (Dex, $10 \mu \mathrm{M}$ ) for 48 hours. [A]. iNOS, PGES2 and COX-2 were revealed by using specific antibodies (upper panel). GAPDH worked as a loading control. Quantification of target protein was calculated by a densitometer (lower panel). [B]: The amounts of NO were evaluated using fluorescent NO indicator DAFFM DA for labeling $30 \mathrm{~min}$. Micrographs were taken by a confocal microscope (upper panel), Bar $=100$ $\mu \mathrm{m}$. Quantification of NO production was calculated by ZEN2009 software (lower panel). Here, dexamethasone (Dex) at $10 \mu \mathrm{M}$ served as a positive control. The values are expressed as the fold of change ( $x$ basal) to blank control, in Mean \pm SEM. ${ }^{\star \star \star} p<0.001$ was considered as significant result compared to the blank group (no drug). ${ }^{\wedge} p<0.05,{ }^{\wedge \wedge} p<0.01$ and ${ }^{\wedge \wedge \wedge} p<0.001$ were categorized as significant result compared to the LPS group. 

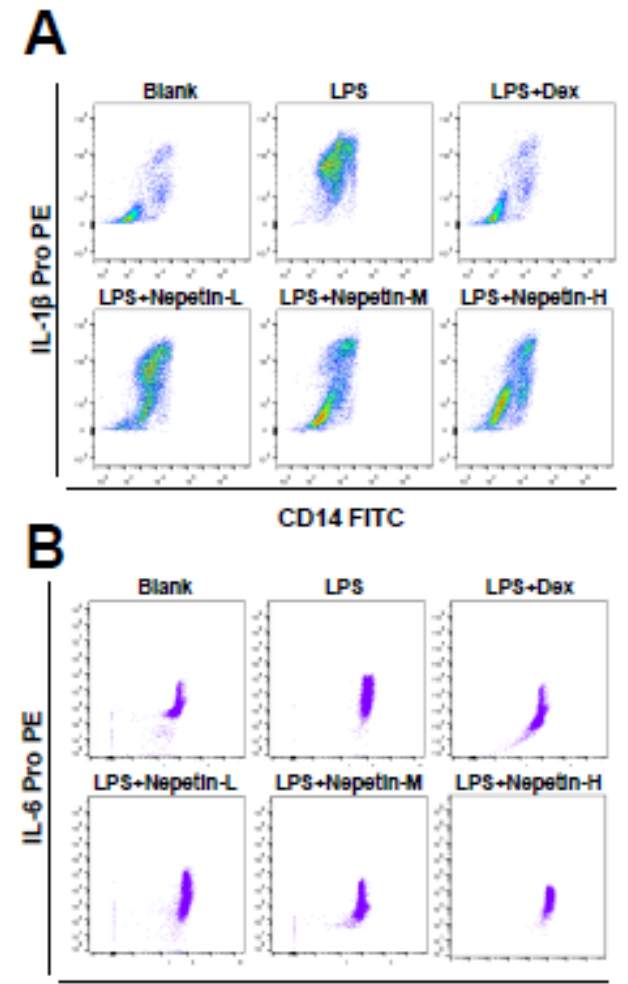

CD14 FITC

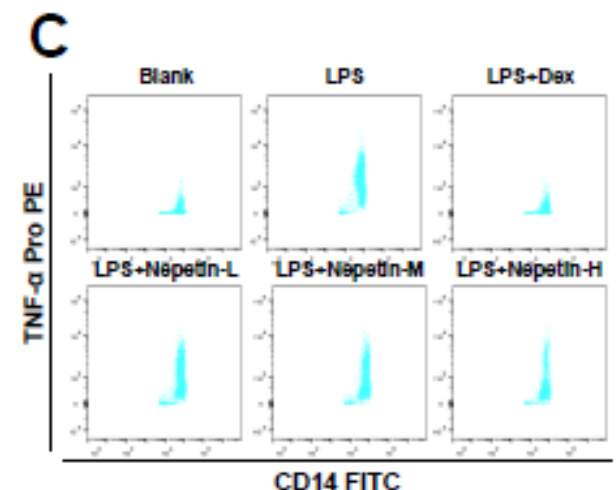

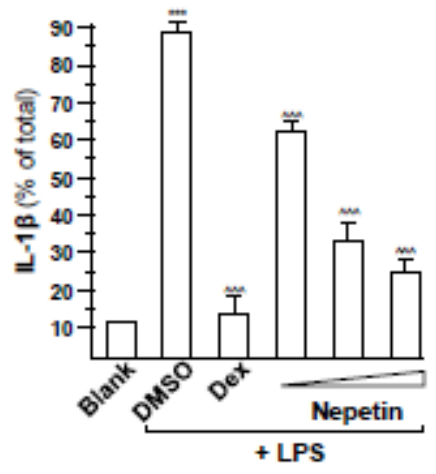
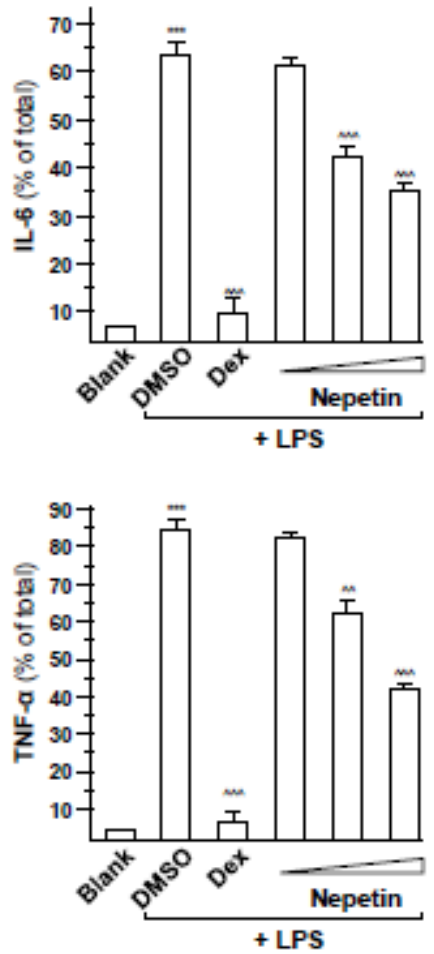

\section{Figure 3}

Nepetin suppreses cytokine production. Cytokine production was detected by flow cytometry. Cultured HaCaT cells was treated with LPS $(1 \mu \mathrm{g} / \mathrm{mL})$ for 24 hours and then applied with a series of nepetin $(0.3$ $\mu \mathrm{M}$ at $\mathrm{L}, 1 \mu \mathrm{M}$ at $\mathrm{M}$ and $3 \mu \mathrm{M}$ at $\mathrm{H}$ as indicated) for another 48 hours as in Figure 1. The dual parametric dot plots combining CD-14-FITC and IL-1 $\beta$ Pro PE [A] or IL-6 Pro PE [B] or TNF-a [C] showed cytokine production (left panel). The quantification analysis was performed by Flowjo v7.6 software (right panel). Dexamethasone (Dex) at $10 \mu \mathrm{M}$ was served as a positive control. The values are expressed as the percentage of total cell number, in Mean \pm SEM. ${ }^{\star \star *} p<0.001$ was considered as significant result compared to the blank group (no drug). ${ }^{\wedge} p<0.01$ and ${ }^{\wedge \wedge \wedge} p<0.001$ were categorized as significant result compared to the LPS group. 


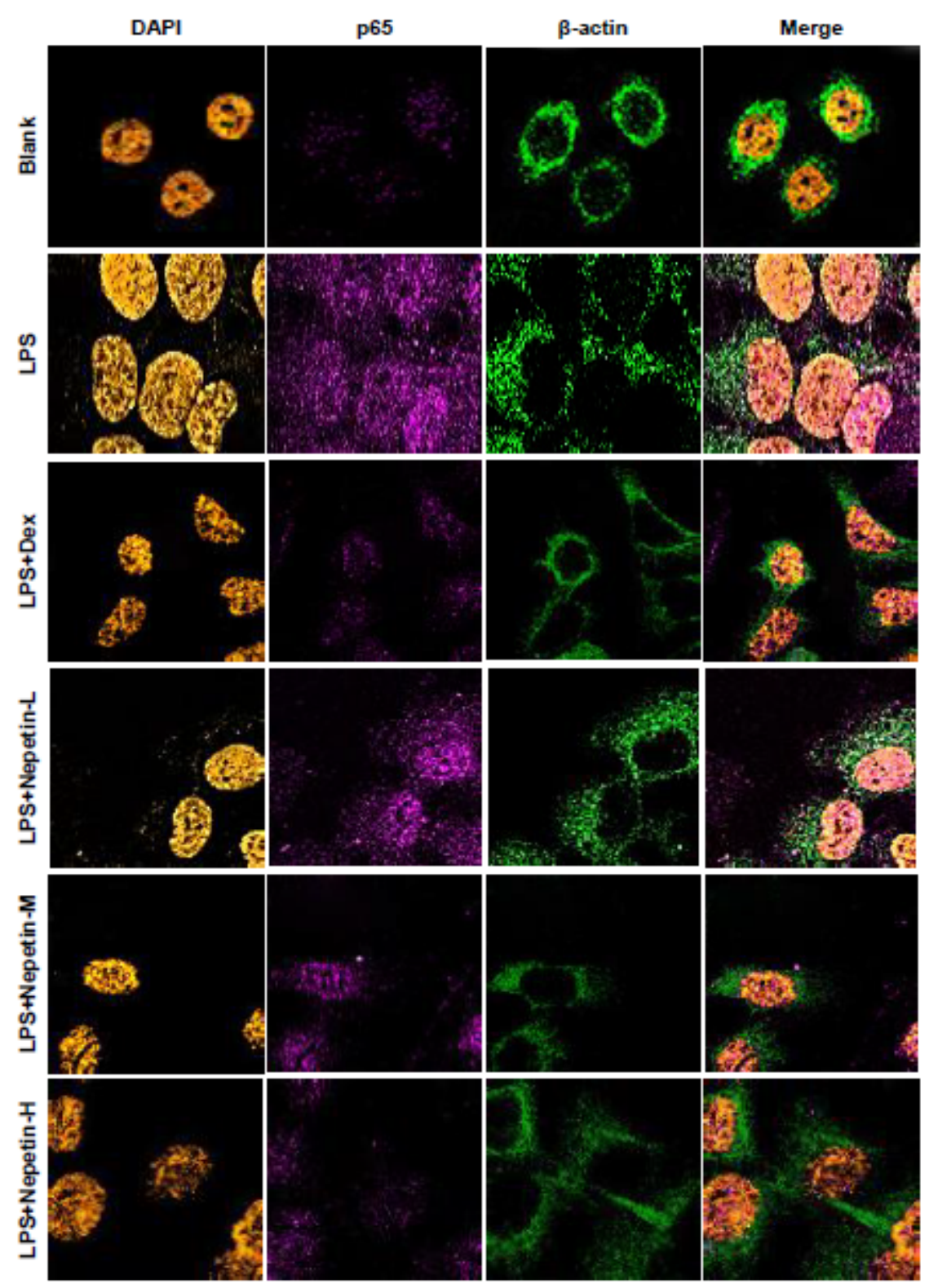

Figure 4

Nepetin blocks the translocation of NF-KB The translocation of NF-KB from cytosol to nucleus was suppressed upon different concentration of nepetin in LPS-induced HaCaT cells for 48 hours.

Dexamethasone (Dex; $10 \mu \mathrm{M}$ ) acted as a positive control. p65 nuclear localization (purple color) was revealed by confocal microscopy. Nuclei were stained by DAPI as shown in orange color. Beta-actin acted as internal control as labeled by green. LPS $(1 \mu \mathrm{g} / \mathrm{mL})$ mimicked the atopic dermatitis environment. Representative photos are shown. Bar $=10 \mu \mathrm{m}$. 

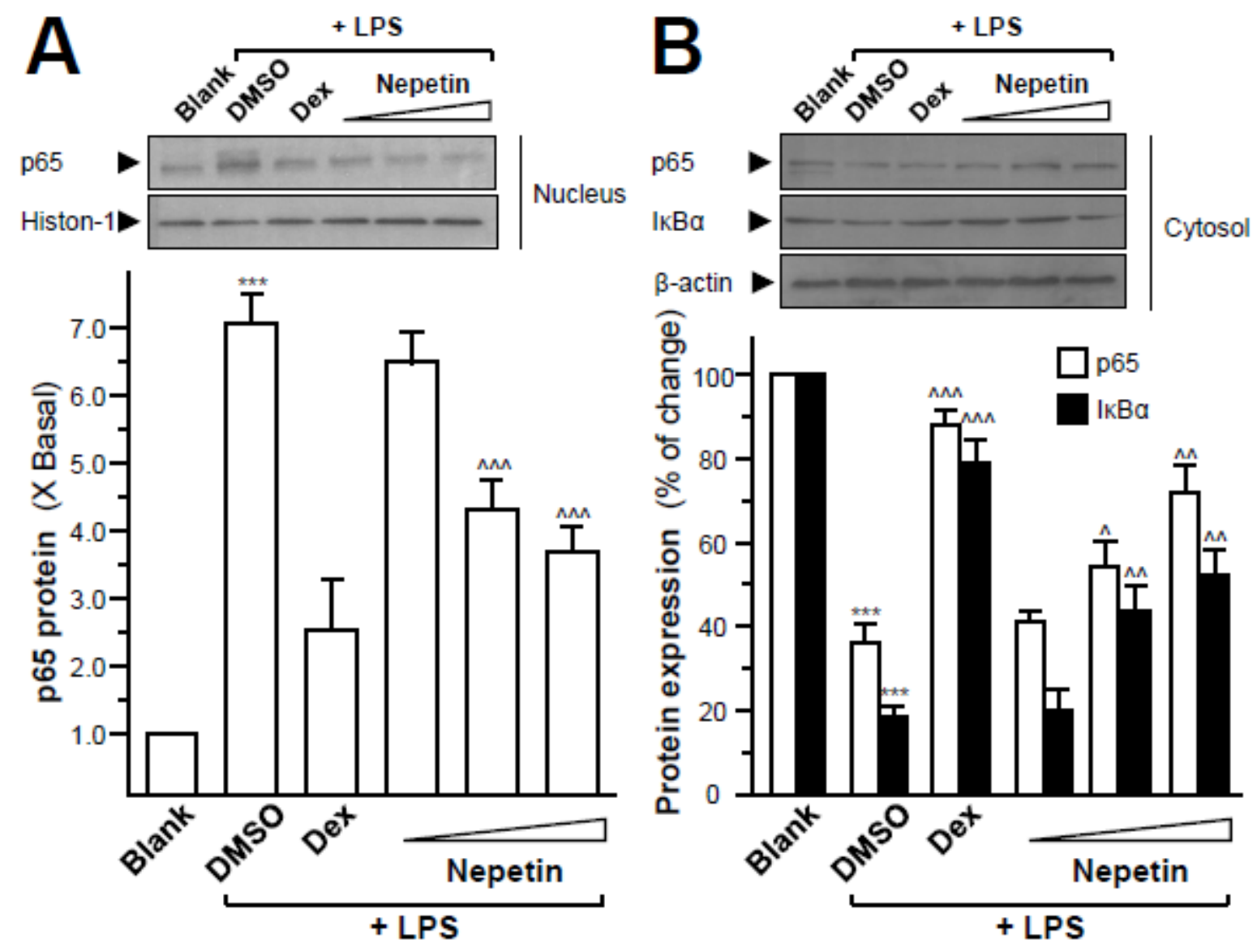

\section{Figure 5}

Nepetin abolishes LPS-induced NF-KB transduction Cultured HaCaT cells were pre-treated with LPS (1 $\mu \mathrm{g} / \mathrm{mL}$ ) for 24 hours and then applied with different dosage of nepetin or dexamethasone (Dex;10 $\mu \mathrm{M})$ for 48 hours, as in Figure 1. The nuclear (A) and cytosolic (B) proteins were isolated. The protein levels of p65 and IкBa were detected by Western blot. Histon-1 or $\beta$-actin served as a loading control (upper panel). Quantification of target protein was calculated by a densitometer (lower panel). The values are expressed as the fold of changes ( $x$ basal) or the percentage of change in corresponding blank control, in Mean \pm SEM. Statistically significant changes were classified as significant $\left({ }^{* \star *}\right)$ where $p<0.001$ as compared to the blank group (no drug). ${ }^{\wedge} p<0.05,{ }^{\wedge \wedge} p<0.01$ and ${ }^{\wedge \wedge} p<0.001$ were categorized as significant result compared to the LPS group. 

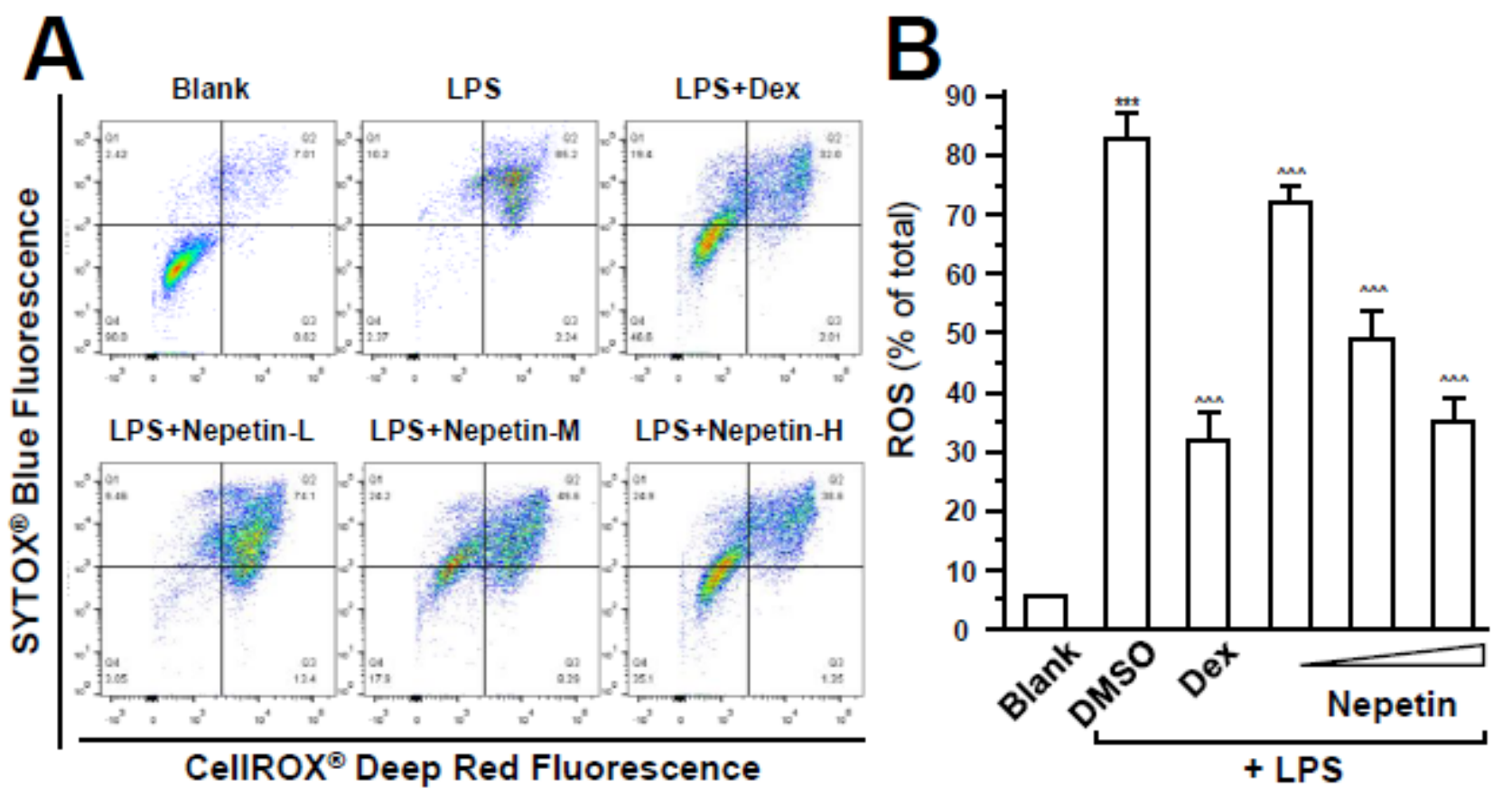

Figure 6

Nepetin attenuates ROS formation HaCaT cells were seeded and pre-treated with LPS for 24 hours then applied with nepetin or dexamethasone (Dex; $10 \mu \mathrm{M})$ for another 48 hours, as in Figure 1. ROS formation was detected by FACSAria [A] and analyzed by Flowjo software [B]. The values are expressed as percentage of total cell number, in Mean \pm SEM. $* \star * p<0.001$ was considered as significant result compared to the blank group (no drug). ${ }^{\wedge \wedge \wedge} p<0.001$ was categorized as significant result compared to the LPS group.

\section{Supplementary Files}

This is a list of supplementary files associated with this preprint. Click to download.

- SupplementaryFig.pdf 